Annuaire suisse de politique de développement

16 | 1997

Environnement et développement, 5 ans après Rio

\title{
1. Situation économique
}

\section{(2) OpenEdition}

1 Journals

Édition électronique

URL : http://journals.openedition.org/aspd/787

DOI : 10.4000/aspd.787

ISSN : 1663-9669

Éditeur

Institut de hautes études internationales et du développement

\section{Édition imprimée}

Date de publication : 1 mars 1997

Pagination : $3-11$

ISSN : 1660-5934

\section{Référence électronique}

«1. Situation économique », Annuaire suisse de politique de développement [En ligne], 16 | 1997, mis en ligne le 06 août 2012, consulté le 08 septembre 2020. URL : http://journals.openedition.org/aspd/787 ; DOl : https://doi.org/10.4000/aspd.787

(c) The Graduate Institute I Geneva 


\section{SITUATION ÉCONOMIQUE}

e chapitre a pour objectif de décrire succinctement la situation économique en 1995 par grandes régions géographiques et de replacer les relations entre la Suisse et les pays en développement du Sud et d'Europe centrale et orientale dans le contexte de l'évolution économique de ces pays.

Alors que la reprise économique dans les pays industrialisés occidentaux s'était imposée sur un large front en 1994, la conjoncture s'est à nouveau considérablement affaiblie au cours du premier semestre 1995. En 1995, la dynamique a été clairement plus forte à l'extérieur de la zone de l'OCDE. Des exportations vigoureuses et une nette hausse de la demande intérieure associées à des investissements directs étrangers toujours plus élevés maintiennent la croissance à un niveau très élevé de $8 \%$ dans les économies dynamiques du sud-est asiatique.'

Tableau $\mathbf{n}^{\circ} \mathbf{1}$

Taux de croissance annuel du produit national brut (en \%)

\begin{tabular}{lrrrr}
\hline & $\mathbf{1 9 9 2}$ & $\mathbf{1 9 9 3}$ & $\mathbf{1 9 9 4}$ & $\mathbf{1 9 9 5}$ \\
\hline Monde & $\mathbf{2 . 4}$ & $\mathbf{2 . 4}$ & $\mathbf{3 . 7}$ & $\mathbf{3 . 5}$ \\
\hline & & & & \\
\hline PI a) & 1.7 & 0.8 & 2.8 & 2.1 \\
\hline PVD $^{\text {b) }}$ & 6.3 & 6.2 & 6.4 & 5.9 \\
\hline Pays en transition c) & -14.7 & -8.5 & -8.8 & -1.3 \\
\hline
\end{tabular}

a) Pays industrialisés.

b) Pays en voie de développement.

c) Ex-URSS, Bulgarie, ex-Tchécoslovaquie, Roumanie, Pologne, Hongrie.

Source : F.M.I. Rapport annuel 1996.

Le commerce mondial est demeuré extrêmement dynamique au cours de l'année écoulée. En 1995, les exportations mondiales de marchandises ont marqué une solide progression en volume, tandis que la valeur combinée du commerce international de marchandises et de services franchissait pour la première fois la barre des 6'000 milliards de dollars². L'augmentation de son volume d'environ $10 \%$ est due principalement à une demande supérieure à la moyenne enregistrée dans les régions autres que les pays industrialisés occidentaux. Par contre, les échanges entre pays de l'OCDE ont perdu quelque peu de leur élan à cause du ralentissement conjoncturel. L'exceptionnelle dynamique des échanges commerciaux des pays d'Europe centrale et orientale est particulièrement frappante : les volumes d'exportation et d'importation des principaux pays de cette zone (Pologne, Hongrie, Slovaquie et République Tchèque) ont en effet augmenté d'environ $20 \%$ en $1995 .{ }^{3}$ Malgré la récession dans certains pays d'Amérique

\footnotetext{
Rapport sur la politique économique extérieure 95/1+2 (Message $\mathrm{n}^{\circ} 95.091$ ).

Focus, Bulletin d'information de l'OMC mai 1996, $\mathrm{n}^{\circ} 10$.

${ }^{3}$ Rapport sur la politique économique extérieure 95/1+2 (Message $n^{\circ} 95.091$ ).
} 
latine, le commerce extérieur des pays en développement s'est accéléré, non seulement du fait des relations au sein de ce groupe, mais aussi en raison de la pénétration toujours plus grande de leurs importations sur les marchés des pays industrialisés et des délocalisations opérées par ces derniers. ${ }^{4}$

Tableau $n^{\circ} 2$

Commerce mondial des marchandises par région, 1994

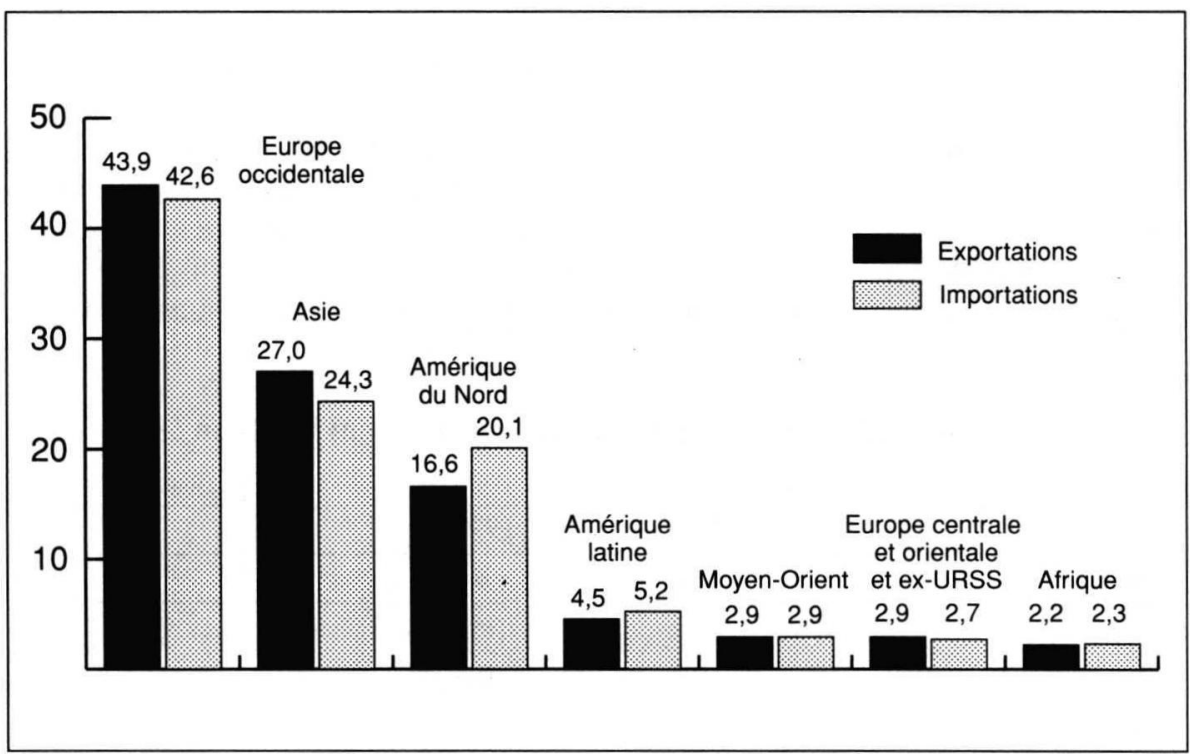

Source : Le commerce international, Tendances et statistiques, 1995, Organisation mondiale du commerce, nov. 1995, p. 7.

L'amélioration sensible des résultats commerciaux de l'Europe occidentale et l'accélération du commerce en Asie, en Amérique du Nord et en Amérique latine, ainsì qu'en Europe centrale et orientale et dans l'ex-URSS, sont à l'origine de l'augmentation de $9,5 \%$ en volume du commerce mondial de marchandises.

\section{SITUATION ÉCONOMIQUE EN 1995 PAR RÉGIONS}

\subsection{PAYS INDUSTRIALISÉS}

La croissance économique s'est ralentie l'an dernier dans les pays industrialisés. Si la chronologie et les causes de ce ralentissement ont varié selon les cas, la plupart des pays ont présenté une caractéristique commune : la faible consommation privée, les ménages hésitant à réduire leur épargne, du fait notamment de la précarité de l'emploi. En outre l'anticipation d'un nouvel effort d'assainissement des finances publiques a pu avoir un effet défavorable (qui reste à l'ordre du jour de nombreux pays de l'OCDE). En Amérique du Nord, le refroidissement

${ }^{4}$ Banque des règlements internationaux, 66 rapport annuel, Bâle : BRI juin 1996, p. 31. 
attendu de l'activité économique aux Etats-Unis et les retombées de la crise mexicaine ont ralenti l'activité économique. Au Japon, la forte appréciation du yen et les difficultés croissantes sur les marchés financiers ont provoqué une perte de confiance des investisseurs et des consommateurs. En conséquence, la demande intérieure a stagné et le PIB réel a même légèrement reculé au cours du premier semestre. En Europe occidentale, l'évolution de la conjoncture a été freinée par les effets retardés des taux réels élevés, mais aussi par des mesures fiscales, en Allemagne notamment. Au total, la croissance de la zone OCDE s'est ralentie en passant d'un taux d'environ 3\% en 1994 à 2,3\% en 1995.

La situation sur le marché du travail a reflété l'évolution de la demande globale et a connu d'amples variations en cours d'année et d'un pays à l'autre. Aux Etats-Unis, le chômage a reculé jusqu'à s'inscrire à un taux généralement considéré comme son taux naturel (taux compatible avec une inflation stable) à 5,5\%, la situation ne s'est pas détériorée au Japon (3,3\%). Dans de nombreux pays d'Europe occidentale, le taux de chômage approchait 10\% en 1996, taux plus élevé qu'en $1992 .^{5}$

La croissance économique de la Suisse ${ }^{6}$, déjà médiocre l'année précédente, s'est à nouveau affaiblie au cours de l'année écoulée. Différents facteurs semblent avoir contribué à cette situation : la demande intérieure (des ménages et du secteur public) a perdu de sa vigueur et dans le domaine de l'économie extérieure, la croissance a été affectée par une nouvelle appréciation du franc. La croissance de l'économie suisse a encore été très modérée pour une deuxième année de reprise économique. En 1994 déjà, l'augmentation du PIB réel était plutôt décevante puisqu'elle n'atteignait que 1,0\%, en 1995 la croissance ne devrait s'élever qu'à $0,1 \%$ environ. Cette faiblesse persistante de la croissance contraste avec la reprise économique observée dans les pays de l'Union européenne, qui ont enregistré un taux de croissance moyen de 2,8\% en 1994 et de 2,5\% en 1995. L'absence de fortes pressions inflationnistes et une réduction de taux d'intérêt dans la plupart des pays constituent des conditions favorables à une reprise lente mais continue de la croissance économique dans la zone OCDE. Les impulsions provenant de la demande des autres régions demeurent un soutien considérable à la croissance. L'OCDE s'attend, pour les deux prochaines années, à une reprise progressive de la croissance dans les pays industrialisés occidentaux $(2,1 \%$ en 1996 et $2,5 \%$ en 1997). De plus, les taux de croissance dans les trois grandes régions - Amérique du Nord, Japon et Europe occidentale - devraient se rapprocher de manière constante, ce qui devrait contribuer à réduire les risques monétaires. Cependant, même après une période de croissance favorable, le taux de chômage en Europe se situera encore au-dessus de $10 \%$.

\subsection{PAYS EN DÉVELOPPEMENT}

La situation économique du monde en développement, y compris dans les économies en transition, a connu une évolution favorable en 1995. Mais comme d'habitude, cette croissance a été très inégalement distribuée et ce sont les pays

5 OCDE, Perspectives économiques de l'OCDE 1996, Paris : juin 1996, p. 7.

${ }^{6}$ Rapport sur la politique économique extérieure 95/1+2 (Message $\mathrm{n}^{\circ} 95.091$ ), pp. 112 et suivante. 
les moins avancés (PMA) qui en ont le moins bénéficié. En Asie du Sud-Est, la croissance est restée très soutenue, tandis qu'en Europe orientale et en Afrique subsaharienne les progrès de 1994 ont été consolidés. Même en Amérique latine la croissance est restée satisfaisante dans la plupart des pays ; l'Argentine et le Mexique ont constitué à cet égard deux exceptions importantes durant la majeure partie de l'année, ce qui était prévisible compte tenu de la crise mexicaine. Dans les économies en développement, l'inflation s'inscrit souvent à des niveaux généralement excessifs, alimentés bien souvent par des déficits budgétaires, mais une volonté officielle de contenir la hausse des prix se manifeste de plus en plus et des progrès sensibles ont été obtenus dans certains pays (voir tableau 4).

Tableau $n^{\circ} 3$

Taux de croissance du PNB annuel des pays en voie de développement

\begin{tabular}{lrrrr}
\hline & $\mathbf{1 9 9 2}$ & $\mathbf{1 9 9 3}$ & $\mathbf{1 9 9 4}$ & $\mathbf{1 9 9 5}$ \\
\hline Ensemble en \% & $\mathbf{6 . 3}$ & $\mathbf{6 . 2}$ & $\mathbf{6 . 4}$ & $\mathbf{5 . 9}$ \\
\hline Afrique & & & & \\
\hline Asie & 0.7 & 0.7 & 2.4 & 3.2 \\
\hline Moyen Orient & 8.7 & 8.9 & 8.8 & 8.4 \\
\hline Amérique latine & 6.2 & 3.7 & 0.7 & 3.7 \\
\hline
\end{tabular}

Remarque : les chiffres de la croissance par grandes régions ne sont pas les mêmes que ceux du rapport BRI, ce qui s'explique par le choix de différents indicateurs selon les institutions financières.

Source : F.M.I. Rapport annuel 1996.

Amérique latine : Le taux de croissance de l'Amérique latine a passé de 4,5\% en 1994 à $0,2 \%$ en 1995 . La production totale des pays de cette région s'est maintenue à l'exception du Mexique et de l'Argentine. A la suite de la crise du peso, le Mexique s'est trouvé confronté à une volatilité de son cours de change et à de vives fluctuations des taux d'intérêt, qui ont créé un climat d'instabilité financière. Le système bancaire a été aux prises avec de graves difficultés et l'inflation est demeurée très élevée. De plus la situation s'est encore aggravée par une diminution de la demande intérieure de 19\%. L'Argentine est le pays qui a le plus subi les contrecoups de la crise mexicaine, la demande intérieure a chuté de $7 \%$. Au Brésil, la politique de stabilisation qui avait abouti à la mise en place du Plan real en juillet 1994 (introduction du real, nouvelle monnaie) a mieux réussi que les réformes monétaires antérieures. Ainsi le plan a contribué à réduire le rythme moyen de hausse des prix sur le continent : entre l'hiver austral de 1994 et celui de 1995 , le taux de l'inflation du pays est passé de 1120 à 25\%, soit la progression la plus faible enregistrée depuis un siècle. Comme le relève le rapport de la $\mathrm{BRI}^{7}$, ce succès peut s'expliquer par trois facteurs: premièrement, par les mesures prises pour s'attaquer dès le départ à l'inertie de l'inflation en freinant l'indexation des salaires sur les prix, deuxièmement par la réduction du déficit budgétaire et enfin par les taux d'intérêt très élevés qui ont attiré les capitaux, provoquant une augmentation notable des réserves de change. Paradoxalement,

${ }^{7}$ Banque des règlements internationaux, 66 rapport annuel, Bâle : BRI juin 1996, p. 47. 
le Plan real a « trop » stimulé la demande intérieure, ce qui a provoqué des déséquilibres, tel le doublement des importations entre les deux premiers semestres de 1994 et 1995. D'autre part, les sorties de capitaux déclenchées par la crise mexicaine ont amputé les réserves internationales du pays. Pour lutter contre ces déséquilibres, les autorités ont imposé des contingents à l'importation, augmenté le droit de douane, laissé glisser le taux de change et durci leur politique monétaire. Vers le milieu de 1995, ces mesures ont réussi à freiner la demande intérieure et à limiter le déficit commercial.

Asie : La production a augmenté à un rythme presque aussi rapide qu'en 1994, malgré le ralentissement en Chine, à Hong Kong, Singapour et Taiwan. L'expansion de l'activité en Asie s'est, une fois encore, centrée sur les exportations. Ces pays ont réalisé de nouvelles percées sur les marchés du monde industriel et le commerce intrarégional s'est développé. Malgré des entrées massives de capitaux et une industrialisation rapide, les pays d'Asie du Sud-Est ont maintenu depuis la fin des années 80 des cours de change vis-à-vis du dollar qui sont restés relativement stables (Thaïlande et Malaisie) ou ont évolué à un rythme remarquablement régulier (Singapour, Indonésie). En Inde, les premiers résultats du processus de libéralisation engagé dès 1991 apparaissent ; la croissance semble avoir été dopée et la production industrielle a augmenté à un rythme supérieur à $10 \%$ depuis le milieu de 1994. En Chine, le programme de stabilisation appliqué depuis le milieu de 1993 a réussi à ralentir quelque peu le rythme de croissance et à réduire l'inflation. Les contrôles administratifs et les restrictions de crédit ont fortement comprimé les dépenses d'investissement. D'autre part, bien que les exportations se soient nettement ralenties, elles ont continué d'augmenter plus vite que les importations, faisant monter l'excédent commercial à plus de 23 milliards de dollars.

Afrique $^{8}$ : Le taux de croissance a été légèrement supérieur à 3\% en 1995. C'est le taux le plus élevé depuis ces six dernières années, mais il ne permet cependant pas d'améliorer de manière significative le niveau de vie. Sur le plan commercial, la croissance en valeur du commerce de l'Afrique $(2,2 \%$ du commerce mondial), bien que positive pour la première fois depuis le début de la décennie, a été faible. Les résultats commerciaux de l'Afrique dépendent en grande partie de trois facteurs économiques : premièrement des prix des produits primaires (y compris le pétrole, les minéraux et les matières premières agricoles); ensuite de la croissance de la production agricole, enfin des résultats de l'Afrique du Sud, qui assure environ un quart du commerce de l'Afrique. En 1994, les exportations de l'Afrique ont beaucoup pâti de la baisse du prix du pétrole. Pour les pays traditionnellement exportateurs de pétrole et de gaz - Algérie, Angola, Libye et Nigeria - les exportations ont donc diminué en valeur. De plus, la sécheresse qui a sévi dans de nombreuses régions de l'Afrique a eu des incidences sur la production agricole, d'où une augmentation de l'importation de produits alimentaires. La valeur des importations de marchandises à l'ensemble de l'Afrique a augmenté de 3\% en 1994. De manière générale, même si les résultats obtenus par l'Afrique à l'exportation se sont légèrement améliorés en 1994 par rapport aux

${ }^{8}$ Pour la rédaction de ce chapitre, nous nous appuyons sur les rapports des grandes organisations internationales (BM, FMI, BRI, OMC, OCDE). Nous avons malheureusement constaté que la situation économique de l'Afrique est tout simplement ignorée par bon nombre de ces rapports. 
Tableau $n^{\circ} 4$

Croissance, inflation

\begin{tabular}{|c|c|c|c|c|c|c|}
\hline \multirow{3}{*}{ Pays/Région } & \multicolumn{3}{|c|}{ PIB réel } & \multicolumn{3}{|c|}{ Prix à la consommation } \\
\hline & \multicolumn{6}{|c|}{ variation annuelle, en $\%$} \\
\hline & $1983-93$ & 1994 & 1995 & $1980-93$ & 1994 & 1995 \\
\hline & moyenne & & & moyenne & & \\
\hline Chine & 9.4 & 11.9 & 10.2 & 8.1 & 24.9 & 17.0 \\
\hline Inde & 5.3 & 6.3 & 6.2 & $8.8^{1}$ & $10.5^{1}$ & $9.3^{1}$ \\
\hline Autres pays d'Asie ${ }^{2}$ & 6.6 & 7.5 & 7.7 & 7.5 & 6.5 & 6.4 \\
\hline Corée & 7.8 & 8.6 & 9.0 & 7.8 & 6.3 & 4.5 \\
\hline Hong Kong & 6.6 & 5.4 & 4.6 & 8.9 & 8.6 & 9.2 \\
\hline Singapour & 7.4 & 10.2 & 8.9 & 2.8 & 3.1 & 1.7 \\
\hline Taïwan & 7.5 & 6.5 & 6.1 & 4.2 & 4.1 & $3 ., 7$ \\
\hline Indonésie & 6.1 & 7.5 & 8.1 & 9.3 & 8.5 & 9.4 \\
\hline Malaisie & 6.6 & 9.2 & 9.5 & 3.7 & 3.7 & 3.4 \\
\hline Philippines & 1.6 & 4.3 & 4.8 & 13.8 & 9.1 & 8.1 \\
\hline Thaillande & 7.7 & 8.5 & 8.6 & 5.4 & 5.1 & 5.8 \\
\hline Amérique latine ${ }^{2}$ & 2.3 & 4.5 & 0.2 & 152.0 & 276.3 & 42.2 \\
\hline Argentine & 1.1 & $\cdot 7.4$ & -4.4 & 284.1 & 4.2 & 3.4 \\
\hline Brésil & 2.0 & 5.8 & 4.2 & 393.4 & 2084.8 & 65.1 \\
\hline Chili & 4.4 & 4.2 & 8.5 & 20.5 & 11.4 & 8.2 \\
\hline Colombie & 3.5 & 5.7 & 5.5 & 24.5 & 23.8 & 21.0 \\
\hline Mexique & 2.3 & 3.5 & -6.9 & 50.1 & 7.0 & 35.0 \\
\hline Venezuela & 1.6 & -2.8 & -2.2 & 25.5 & 60.8 & 59.9 \\
\hline Europe orientale $^{2}$ & -0.5 & 4.2 & 5.5 & 37.6 & 24.2 & 23.2 \\
\hline Hongrie & -0.8 & 5.2 & 7.0 & 61.3 & 32.2 & 27.8 \\
\hline Pologne & -0.2 & 2.6 & 4.8 & 7.1 & 10.0 & 9.1 \\
\hline République tchèque $^{3}$ & -0.2 & 2.6 & 4.8 & 7.1 & 10.0 & 9.1 \\
\hline Fédération de Russie ${ }^{4}$ & 0.1 & -12.6 & -4.0 & $98.1^{5}$ & 307.4 & 197.7 \\
\hline Afrique & 2.1 & 2.0 & 3.2 & 21.6 & 33.9 & 25.8 \\
\hline Afrique du Sud & 1.4 & 2.7 & 3.3 & 14.2 & 9.0 & 8.6 \\
\hline Pays de la zone franc & 1.4 & 1.6 & 3.8 & 4.8 & 25.6 & 16.1 \\
\hline Arabie Saoudite & 1.7 & -0.1 & 1.6 & 0.6 & 0.6 & 5.0 \\
\hline Israël & 3.9 & 6.5 & 7.0 & 73.5 & 12.3 & 10.0 \\
\hline
\end{tabular}

Remarque : Les données pour 1995 sont en partie estimées.

${ }^{1}$ Prix de gros. ${ }^{2}$ Moyenne pondérée des pays cités, sur la base des PIB et PPA de 1990. ${ }^{3}$ Avant 1985, Tchécoslovaquie.

${ }^{4}$ Avant 1986, Union soviétique. ${ }^{5}$ Moyenne pour $1985-1993$.

Sources : Banque mondiale, World Tables; données nationales, in : Banque des règlements internationaux, 66e Rapport annuel, juin 1996, p. 38. 
années précédentes, ils sont restés médiocres. La valeur des exportations s'est accrue de $2 \%$ (moins de $1 \%$ si l'on exclut l'Afrique du Sud), et la part de l'Afrique dans les exportations mondiales a continué à diminuer. Parmi les pays ayant enregistré une croissance positive de la valeur des exportations de marchandises dans les années 90, on peut citer l'Afrique du Sud, le Bénin, l’Égypte, la Guinée-Bissau, le Kenya, Maurice, l'Ouganda et la Tunisie. ${ }^{9}$

Après avoir à peine augmenté en 1994 (0,7\%), la croissance a repris dans les pays en développement du Moyen-Orient, atteignant 3,7\% en 1995. Cette reprise est principalement due à la Turquie, où, après quelques années de récession, la croissance a atteint $7 \%$ en 1995. L'amélioration de la stabilité politique dans la région a aidé à soutenir une légère reprise dans quelques pays, notamment en Israël, au Liban et en Jordanie..$^{10}$ Les résultats commerciaux de la plupart des pays du Moyen-Orient demeurent étroitement liés au prix du pétrole et du gaz naturel sur les marchés mondiaux, et en 1994 la baisse du prix du pétrole a de nouveau limité la croissance du revenu et la demande d'importations. Parmi les pays dont les importations se sont accrues en 1994, on peut citer Chypre, Israël, le Liban, Oman, les Emirats arabes Unis et la Syrie. Le marasme du marché du pétrole a eu également des incidences sur les exportations de marchandises qui ont stagné en valeur (mais augmenté en volume). ${ }^{11}$

\subsection{PAYS EN TRANSITION}

La croissance dans la plupart des pays d'Europe centrale et orientale (PECO), poussée par les exportations et l'investissement, a sans doute atteint son apogée en 1995, mais elle devrait rester robuste en 1996. Dans l'ex-Union soviétique, si la production a généralement continué de décroître en 1995, les signes de reprise deviennent plus tangibles. L'intégration de la région dans l'économie mondiale s'est accélérée avec l'essor du commerce extérieur et l'augmentation des entrées de capitaux. Les réformes structurelles se sont poursuivies, quoique de façon inégale, avec parfois des retours en arrière. Dans la plupart des pays de la région, la croissance s'est améliorée en 1995, avoisinant les 5\% dans les pays les plus avancés (voir tableau 4). Parmi les raisons du dynamisme de la production dans les PECO on peut citer, du côté de l'offre, des gains de productivité considérables (certes à partir d'un niveau réduit) et un redressement dans l'agriculture après une série de mauvaises récoltes. Côté demande, l'expansion a été alimentée par les exportations. Cette expansion a favorisé un redressement de l'emploi. La région a continué à développer ses exportations à un rythme très supérieur à la moyenne mondiale pour la troisième année consécutive. Les importations ont également augmenté à un rythme soutenu, souvent en raison d'achat de biens intermédiaires et de biens d'équipement, notamment dans le contexte de la relocalisation des activités de transformation (sous-traitance par des entreprises de l'Union européenne aux PECO d'une partie de leurs activités de production). A la faveur d'une politique budgétaire relativement prudente, la désinflation s'est

\footnotetext{
${ }^{9}$ Organisation mondiale du commerce, Le commerce international, tendances et statistiques 1995, Genève : OMC, nov. 1995 , p. 12.

${ }^{10}$ Fonds monétaire international, Rapport annuel, Washington : FMI, 1996, p.16.

${ }^{11}$ Organisation mondiale du commerce, Le commerce international, tendances et statistiques 1995, Genève : OMC, nov. 1995, p. 12.
} 
poursuivie dans la plupart des PECO en 1995. Cependant en Russie et en Ukraine, les efforts de stabilisation déployés ont eu un certain effet, mais l'inflation demeure très élevée. La privatisation s'est poursuivie avec détermination, empruntant de nombreuses voies différentes : lorsque l'Etat a conservé des blocs d'actions, le risque d'une politisation de la gestion demeure, en revanche dans les entreprises où les investissements étrangers ont été admis, la restructuration s'est révélée généralement plus efficace. L'intégration croissante dans l'économie mondiale s'est aussi traduite par la libéralisation des opérations courantes et des mouvements de capitaux. Un certain nombre de pays ont déclaré officiellement la convertibilité de leurs monnaies conformément aux prescriptions du FMI. Dans ce processus de participation à l'économie mondiale, la plupart des nouveaux Etats indépendants de l'ex-Union soviétique (NEI) restent manifestement à la traîne, certains ayant opté pour une stratégie plus autocentrée et cherchant à renouer entre eux des relations basées sur des critères autres que de marché. ${ }^{12}$

\subsection{RAPPORTS D'ORGANISATIONS INTERNATIONALES}

\section{$\square$ Rapport de la Banque mondiale 1996 sur les économies en transition}

Dix-neuvième du nom, l'édition de 1996 du Rapport sur le développement ${ }^{13}$ dans le monde traite du passage à l'économie de marché des pays à économie planifiée, en mettant l'accent sur les pays d'Europe centrale et orientale, les nouveaux Etats indépendants de l'ex-Union soviétique, la Chine et le Vietnam. Selon le Rapport ${ }^{14}$, les pays en transition ont fait en peu de temps d'énormes progrès vers l'économie de marché et s'ils persistent dans leurs efforts, ils pourront parvenir à une croissance vigoureuse, à la stabilité financière et à des niveaux de vie plus élevés. Le Rapport énumère une série de pronostics et de conclusions :

- Même lorsque les assises de l'économie de marché sont encore fragiles, l'application de politiques cohérentes, la libéralisation des marchés et l'implantation de nouvelles entreprises, de même qu'une stabilité relative des prix, permettent de beaucoup progresser.

- L'opposition entre thérapie de choc et réforme graduelle n'aide guère l'analyste. Ce qui compte c'est l'ampleur des réformes engagées et la cohérence avec laquelle elles sont appliquées.

- Des droits de propriété bien définis sont nécessaires pour que les forces du marché puissent jouer à plein, ce qui passe, à terme, par une généralisation de la propriété privée.

- Une refonte de la politique sociale pour faire reculer la pauvreté et tenir compte des répercussions des réformes sur les jeunes et sur les personnes âgées doit accompagner la progression vers l'économie de marché.

- Il est crucial de réformer les institutions juridiques et financières, et les administrations publiques elles-mêmes.

${ }^{12}$ OCDE, Perspectives économiques de l'OCDE 1996, Paris : OCDE, juin 1996.

${ }^{13}$ Un résumé du rapport de la Banque mondiale sur les économies en transition se trouve sur Internet à l'adresse : http ://www.worldbank.org/

${ }^{14}$ Banque mondiale, De l'économie planifiée à l'économie de marché, Rapport sur le développement dans le monde 1996. ch. 10 « Bilan et perspectives", Washington : Banque mondiale, p. 170. 
La transition est loin d'être achevée et le processus ne cesse d'exercer une influence profonde sur le plan social, politique et stratégique. Le retour à un système d'économie planifiée n'est guère probable, mais la stagnation à long terme et une pauvreté croissante ne peuvent être exclues dans certains cas.

\section{Rapport mondial sur le développement humain, PNUD, 1996}

Comme les précédents rapports, celui de cette année part du principe que le développement humain est une fin, la croissance économique n'étant qu'un moyen. L'élément central de ce rapport est constitué par l'indicateur du développement humain (IDH) qui classe les pays en fonction de l'espérance de vie, du niveau d'instruction et de la parité du pouvoir d'achat. Cette année un nouvel indicateur a été introduit pour rendre compte des aspects cachés de la pauvreté : l'absence des capacités élémentaires nécessaires pour progresser sur l'échelle du développement humain et sortir de la pauvreté économique. Cet indicateur, appelé indicateur de pénurie de capacités (IPC), montre que nombre de pays manquent davantage de capacités que leur niveau de pauvreté économique le laisserait supposer.

Voici quelques-unes des conclusions de ce rapport :

- Le recul ou la stagnation économique touche 89 pays depuis 10 ans, réduisant les revenus de plus d'un quart de la population mondiale.

- Sur 46 pays qui ont effectivement connu la croissance économique, 19, soit $40 \%$, ont souffert d'une réduction des opportunités d'emplois ou d'une croissance sans création d'emplois.

- Aucun pays ne peut conserver à long terme un niveau de croissance économique élevé si son développement humain ne repose pas sur des bases solides.

Comme toujours, le Rapport mondial sur le développement humain propose des mesures, à la fois à l'échelle nationale et au sein de la communauté internationale, pour aider à mettre en place une croissance durable et à vaincre la pauvreté.

\section{SOURCES}

Rapport sur la politique économique extérieure 95/1+2, in $F F$, vol. 1, $\mathrm{n}^{\circ} 9,5$ mars 1996, (Message $\mathrm{n}^{\circ} 95.091$ ).

Banque des règlements internationaux, 66 rapport annuel, Bâle : BRI, juin 1996.

Organisation mondiale du commerce, Le commerce international, tendances et statistiques 1995, Genève : OMC, nov. 1995.

OCDE, Perspectives économiques de l'OCDE 1996, Paris : OCDE, juin 1996.

Fonds monétaire international, Rapport annuel, Washington : FMI, septembre 1996.

Programme des Nations Unies pour le développement, Rapport sur le développement humain 1996, PNUD, juillet 1996.

Banque mondiale, De l'économie planifiée à l'économie de marché, Rapport sur le développement dans le monde 1996, Washington : Banque mondiale, juin 1996.

Banque nationale suisse, $88^{e}$ rapport de gestion 1995, Zurich : BNS, 1996.

L'économie mondiale 1997, CEPII, La Découverte, Paris, 1996.

Focus, Bulletin d'information de l'OMC, mai 1996, $\mathrm{n}^{\circ} 10$.

Journal de Genève, 21 juin 1996 "L'OCDE voit l'Europe renouer avec la croissance », 27 juin 1996 « L'économie mondiale retrouve le sourire avec l'été ", 8 juillet 1996 " Forte croissance américaine au deuxième trimestre ".

Neue Zürcher Zeitung, 5. Februar 1996 «26. Weltwirtschaftsforum in Davos », 21. Juni 1996, «59. Economic Outlook der OECD ». 